\title{
Reproducibility and intercorrelation of graph theoretical measures in structural brain connectivity networks
}

\author{
Timo Roine $^{\mathrm{a}, \mathrm{b}, *}$, Ben Jeurissen ${ }^{\mathrm{a}}$, Daniele Perrone ${ }^{\mathrm{c}}$, Jan Aelterman ${ }^{\mathrm{c}}$, Wilfried \\ Philips $^{c}$, Jan Sijbers ${ }^{\mathrm{a}}$, Alexander Leemans ${ }^{\mathrm{d}}$ \\ ${ }^{a}$ imec-Vision Lab, Department of Physics, University of Antwerp, Antwerp, Belgium \\ ${ }^{b}$ Turku Brain and Mind Center, University of Turku, Finland \\ ${ }^{c}$ Ghent University-imec/Image Processing and Interpretation, Ghent, Belgium \\ ${ }^{d}$ Image Sciences Institute, University Medical Center Utrecht, Utrecht, The Netherlands
}

\begin{abstract}
Diffusion-weighted magnetic resonance imaging can be used to non-invasively probe the brain microstructure. In addition, recent advances have enabled the identification of complex fiber configurations present in most of the white matter. This has improved the investigation of structural connectivity with tractography methods. Whole-brain structural connectivity networks, or connectomes, are reconstructed by parcellating the gray matter and performing tractography to determine connectivity between these regions. These complex networks can be analyzed with graph theoretical methods, which measure their global and local properties. However, as these tools have only recently been applied to structural brain networks, there is little information about the reproducibility and intercorrelation of network properties, connectivity weights and fiber tractography reconstruction parameters in the brain. We studied the reproducibility and correlation in structural brain connectivity networks reconstructed with constrained spherical deconvolution based probabilistic streamlines tractography. Diffusion-weighted data from 19 subjects were acquired with $\mathrm{b}=2800 \mathrm{~s} / \mathrm{mm}^{2}$ and 75 gradient orientations. Intrasubject variability was computed with residual bootstrapping. Our findings indicate that the reproducibility of graph theoretical metrics is generally excellent with the exception of betweenness centrality. A reconstruction density of approximately one million streamlines is necessary for excellent reproducibility, but the reproducibility increases further with higher densities. The reproducibility decreases, but only slightly, when switching to a higher order in constrained spherical deconvolution. Moreover, in binary
\end{abstract}


networks, using sufficiently high threshold values improves the reproducibility. We show that multiple network properties and connectivity weights are highly intercorrelated. The experiments were replicated by using a test-retest dataset of 44 healthy subjects provided by the Human Connectome Project. In conclusion, our results provide guidelines for reproducible investigation of structural brain networks.

Keywords: Diffusion magnetic resonance imaging, tractography, constrained spherical deconvolution, connectome, reproducibility

\section{Introduction}

Diffusion-weighted (DW ${ }^{1}$ magnetic resonance imaging (MRI) can be used to noninvasively investigate the microstructural properties of the brain. Diffusion in fiber tracts is anisotropic, i.e. larger parallel to the tract than perpendicular to it, which enables the reconstruction of neural pathways in the brain with fiber tracking (Basser et al., 2000; Jeurissen et al., 2017). Diffusion tensor imaging (DTI) is traditionally the most common method used for the analysis of DW-MRI data (Basser et al., 1994a b). However, its major

\footnotetext{
${ }^{*}$ Corresponding author:

Timo Roine

imec-Vision Lab, Department of Physics

University of Antwerp

Universiteitsplein 1, Building N

2610 Wilrijk (Antwerp), Belgium

Email address: timo.roine@utu.fi (Timo Roine)
}

${ }^{1}$ Abbreviations: ACT: anatomically constrained tractography, BC: betweenness centrality, CC: clustering coefficient, $\mathrm{CoV}$ : coefficient of variation, CSD: constrained spherical deconvolution, DG: degree, DTI: diffusion tensor imaging, DW: diffusion-weighted, DWI: diffusion-weighted image, EPI: echo-planar imaging, FA: fractional anisotropy, FIRST: FMRIB Integrated Registration and Segmentation Tool, fODF: fiber orientation distribution function, FOV: field of view, FSL: FMRIB Software Library, GM: gray matter, HCP: The Human Connectome Project, ICC: intraclass correlation coefficient, LE: local efficiency, MPRAGE: magnetization-prepared rapid gradient-echo, MRI: magnetic resonance imaging, nCC: normalized clustering coefficient, $\mathrm{nCPL}$ : normalized characteristic path length, nGE: normalized global efficiency, NEX: number of excitations, SH: spherical harmonics, SIFT: spherical deconvolution informed filtering of tractograms, STR: strength, SW: small-worldness, TE: echo time, TI: inversion time, TR: repetition time, WM: white matter 
limitation is the inability to correctly describe complex fiber configurations such as crossing fibers, present in the majority of white matter (WM) (Jeurissen et al., 2013; Vos et al., 2011). This limitation dramatically impairs the investigation of structural brain connectivity. However, novel analysis methods, such as constrained spherical deconvolution (CSD), are able to estimate the full fiber orientation distribution function (fODF) (Tournier et al., 2004, 2007). This allows for tractography to continue through regions with complex fiber configurations (Tournier et al., 2007, 2012), which enables a more accurate investigation of structural connectivity in vivo (Farquharson et al., 2013; Kristo et al., 2013; Tournier et al., 2008).

Whole-brain structural connectivity networks, or connectomes, are constructed by first parcellating the gray matter (GM) of the brain and then performing whole brain tractography to determine connectivity weights between each pair of regions (Bullmore and Sporns, 2009, Hagmann et al., 2008). The subsequent analysis of these networks can be performed with graph theoretical tools, originally developed for other applications, such as social networks (Bullmore and Sporns, 2009; Rubinov and Sporns, 2010). Graph theoretical analysis produces global and local metrics describing the integration, segregation and centrality of the networks (Rubinov and Sporns, 2010). The reliability of these metrics in structural brain networks has recently been investigated in several studies (Andreotti et al., 2014; Bassett et al., 2011; Bastiani et al., 2012; Buchanan et al., 2014; Cheng et al., 2012; Dennis et al., 2012; Dimitriadis et al., 2017; Drakesmith et al., 2015; Duda et al., 2014; Owen et al., 2013; Parker et al., 2014; Rodrigues et al., 2013; Smith et al., 2015a; Vaessen et al., 2010; Zalesky et al., 2016; Zhong et al., 2015). However, most of these studies were performed with DTI-based tractography (Andreotti et al., 2014; Bassett et al., 2011; Cheng et al., 2012; Duda et al., 2014; Vaessen et al., 2010) lacking the ability to identify complex fiber configurations. Moreover, none of the previous studies has specifically investigated the reproducibility of different network metrics, connectivity weights or reconstruction parameters for CSD-based tractography using high b-value acquisitions, which is becoming one of the more popular strategies to investigate brain connectivity.

The effects of various tractography algorithms on the network properties were investigated by Bastiani et al. (2012), and are thus outside the scope of this article. Bastiani et al. (2012) performed tractography with global and local, deterministic and probabilistic, and DTI, CSD and diffusion orientation distribution function based algorithms, and analyzed the 
effects of different tractography algorithms on the network properties using DW data acquired with $\mathrm{b}=3000 \mathrm{~s} / \mathrm{mm}^{2}$ and 120 gradient directions. They reported that the choice of tractography method and parameters had a large effect on network density and concluded that multiple fiber direction models should be preferred over single direction models, probabilistic over deterministic, and global over local tractography methods. In addition, Parker et al. (2014) compared two state-of-the-art pipelines for analysis of DW data with $\mathrm{b}=1000 \mathrm{~s} / \mathrm{mm}^{2}$ and 60 gradient orientations. The first pipeline included a CSD-based and the second a "ball and sticks" model based tractography approach. The authors found a highly robust consensus network between these two pipelines.

To the authors' knowledge, only six studies have analyzed the reproducibility of graph theoretical analysis in structural connectomes constructed with recent methods capable of identifying complex fiber configurations (Buchanan et al., 2014, Dennis et al., 2012; Dimitriadis et al., 2017; Owen et al., 2013, Smith et al., 2015a: Zhong et al., 2015). Owen et al. (2013) investigated structural network metrics in ten subjects between two scan sessions and found the test-retest reliability to be good-to-excellent. They performed probabilistic tractography with the "ball and sticks" model (Behrens et al., 2007), estimating up to two fiber orientations per voxel. The data were acquired with a b-value of $1000 \mathrm{~s} / \mathrm{mm}^{2}$ and 30 gradient directions. Buchanan et al. (2014) studied the reproducibility in data with $\mathrm{b}=1000 \mathrm{~s} / \mathrm{mm}^{2}$ and 64 gradient orientations acquired from ten subjects with an interval of a few days. They reported that reproducibility was improved by seeding from WM in comparison to GM, and by applying the probabilistic "ball and sticks" tractography instead of deterministic DTI tractography. Dennis et al. (2012) studied the test-retest reliability of data acquired three months apart from 26 adults with $\mathrm{b}=1159 \mathrm{~s} / \mathrm{mm}^{2}$ and 94 gradient directions, and analyzed with q-ball imaging (Tuch, 2004) and tractography based on Hough transform (Aganj et al., 2011). They concluded that the reproducibility was highest for network modularity, and that the mean clustering coefficient had higher reliability than characteristic path length or global efficiency for the lower network sparsities. Smith et al. (2015a) investigated the effect of spherical deconvolution informed filtering of tractograms (SIFT) (Smith et al., 2013, 2015b) on the reproducibility of graph theoretical metrics produced with CSD-based probabilistic tractography. They acquired data with 60 gradient orientations and $b=3000 \mathrm{~s} / \mathrm{mm}^{2}$ from eight subjects, one of which underwent eight repetitions of the scan sequence with subject reposition between each acquisi- 
tion. They concluded that SIFT produced metrics that are both more reproducible and more biologically representative for the WM connectivity. Zhong et al. (2015) investigated the test-retest reproducibility of DTI- and "ball and sticks" based tractography algorithms and different networks weights in DW data acquired from 57 adults scanned twice within a 6-week interval with $\mathrm{b}=1000 \mathrm{~s} / \mathrm{mm}^{2}$ and 30 gradient orientations. They reported a higher reproducibility of networks weighted with the probability of connectivity than with fiber density or fractional anisotropy, and that the reproducibility of binary networks was the lowest. Dimitriadis et al. (2017) found that six out of nine tested network weighting strategies resulted in high reproducibility in a sample of five subjects imaged five times with 30 gradient directions and $\mathrm{b}=1200 \mathrm{~s} / \mathrm{mm}^{2}$, and analyzed with the damped Richardson Lucy spherical deconvolution (Dell'Acqua et al., 2010).

Apart from Smith et al. (2015a), none of the previous research analyzed the reproducibility of graph theoretical metrics produced from high b-value data with CSD-based tractography. Moreover, the effects of reconstruction density and spherical harmonics (SH) order used in CSD have not been investigated earlier in the graph theoretical analysis of structural brain networks. However, CSD-based structural brain networks have already been used in the investigation of, for example, autism (Roine et al. 2015b), preterm children (Thompson et al., 2016), bipolar disorder (Forde et al., 2015), Huntington's disease (Odish et al., 2015) and traumatic brain injury (van der Horn et al., 2016).

In this study, we investigated the reproducibility and intercorrelation of different global and local graph theoretical metrics, network weights and reconstruction parameters in structural brain networks by applying CSDbased probabilistic tractography to state-of-the-art DW data acquired from 19 healthy subjects using $\mathrm{b}=2800 \mathrm{~s} / \mathrm{mm}^{2}$ and 75 gradient directions. These analyses were partially replicated in a test-retest dataset of 44 healthy subjects provided by the Human Connectome Project (HCP) (Van Essen et al., 2013).

\section{Material and methods}

\subsection{Data acquisition}

We acquired high angular resolution DW-MRI data from 19 healthy subjects with a 3T MRI Tim Trio Siemens system and a 32-channel head coil. The participants gave written informed consent to participate in this study 
under a protocol approved by the local ethics committee. A single-shot echoplanar imaging (EPI) sequence was used with repetition time $(\mathrm{TR})=8100$ $\mathrm{ms}$, echo time $(\mathrm{TE})=116 \mathrm{~ms}$ and $2.5 \times 2.5 \times 2.5 \mathrm{~mm}^{3}$ voxel size. The field of view $(\mathrm{FOV})$ was $240 \times 240 \mathrm{~mm}^{2}$, the acquisition matrix was $96 \times 96$, and the number of excitations (NEX) was 1. In the axial direction, 54 slices were imaged with $2.5 \mathrm{~mm}$ thickness and no gap. Diffusion sensitizing gradients along 75 non-collinear directions were applied with $\mathrm{b}=2800 \mathrm{~s} / \mathrm{mm}^{2}$. Ten images without diffusion-weighting $\left(b=0 \mathrm{~s} / \mathrm{mm}^{2}\right)$ were acquired, one of which was acquired with reverse phase-encoding to be able to correct for EPI distortions. High-resolution $\mathrm{T}_{1}$-weighted structural images were acquired with a 3D magnetization-prepared rapid gradient-echo (MPRAGE) sequence (Mugler and Brookeman, 1990) with $\mathrm{TR}=1900 \mathrm{~ms}$, TE $=2.52 \mathrm{~ms}$, inversion time $(\mathrm{TI})=900 \mathrm{~ms}$ and a voxel size of $0.977 \times 0.977 \times 1 \mathrm{~mm}^{3}$ (flip angle $=9^{\circ}$ and NEX = 1). FOV was $250 \times 250 \times 176 \mathrm{~mm}^{3}$ with a $256 \times 256$ $\times 176$ acquisition matrix.

The HCP test-retest dataset (Van Essen et al., 2013), consisting of $\mathrm{T}_{1^{-}}$ weighted and DW-MRI data from 44 healthy subjects, was used to replicate a part of the analyses. The DW-MRI data used in this study consisted of 90 diffusion-weighted images with $\mathrm{b}=3000 \mathrm{~s} / \mathrm{mm}^{2}$ and a resolution of 1.25 $\times 1.25 \times 1.25 \mathrm{~mm}^{3}$. These data were acquired with a test-retest interval of $4.9 \pm 2.4$ months. The HCP acquisition protocol is described in detail in (Feinberg et al., 2010; Moeller et al., 2010; Setsompop et al., 2012; Sotiropoulos et al., 2013a. b; Xu et al., 2012).

\subsection{Preprocessing and segmentation}

The DW data were corrected for subject motion, induced eddy current and EPI distortions using FMRIB Software Library's (FSL) (Jenkinson et al. 2012; Smith et al., 2004) EDDY and TOPUP tools (Andersson et al., 2003; Andersson and Sotiropoulos, 2015, 2016). In the correction procedure, the b-matrices were adjusted to account for rotation components due to subject motion (Leemans and Jones, 2009). Cortical parcellation of the structural $\mathrm{T}_{1}$-weighted images was performed in FreeSurfer (Desikan et al., 2006; Fischl et al., 2004) and combined with the sub-cortical structures segmented with the FMRIB Integrated Registration and Segmentation Tool (FIRST) (Patenaude et al., 2011), as proposed in Smith et al. (2012). Thus, in addition to the parcellation, reliable partial volume fraction maps for all four tissue types (cerebrospinal fluid, cortical and deep GM, and WM) were obtained. The 
structural data were aligned to the DW images using rigid body registration in SPM (Ashburner and Friston, 2003) (http://www.fil.ion.ucl.ac.uk/spm/).

The HCP dataset was provided preprocessed, as described in Glasser et al. (2013), with FSL (Jenkinson et al., 2012), FreeSurfer (Fischl et al., 2004), and the methods described in (Andersson et al., 2003; Andersson and Sotiropoulos, 2015, 2016; Jenkinson et al., 2002; Milchenko and Marcus, 2013).

\subsection{Fiber orientation estimation and probabilistic tractography}

We performed CSD to estimate the full fODF using SH orders from 4 to 12 , by deconvolving the data with a response function describing a single coherently aligned WM fiber population (Tournier et al., 2004, 2007). Probabilistic tractography was performed using the iFOD2 algorithm (Tournier et al. 2010, 2012). Streamlines were seeded from the WM-GM interface, and anatomically constrained tractography (ACT) was used to produce anatomically plausible streamlines originating and terminating in GM (Smith et al., 2012). For most of the experiments, 10 million streamlines were reconstructed for each subject. In addition, in a single experiment, the number of reconstructed streamlines was varied from 10,000 to 100 million (and 10,000 to 10 million in the HCP test-retest sample). However, 100 million streamlines were reconstructed only for two of the subjects due to the long computation time and storage requirements for the bootstrapping data, while this was done for all subjects with the original data. The 100 million streamlines were then filtered to 10 million streamlines using SIFT to maximize their correspondence to the underlying fODFs (Smith et al., 2013; Yeh et al., 2016). The MRtrix3 package (http://www.mrtrix.org) was used for fiber orientation estimation, tractography, SIFT and visualization (Tournier et al., 2012). The step size used in the tractography was $1.25 \mathrm{~mm}$, the fODF amplitude cutoff was 0.1 , and the maximum turning angle was 45 degrees. The range of acceptable streamline lengths was $5 \mathrm{~mm}$ to $250 \mathrm{~mm}$. No backtracking was allowed for ACT.

\subsection{Graph theoretical analysis}

Structural brain networks were constructed by assigning the reconstructed streamlines to the corresponding GM areas according to their endpoints as illustrated in Figure 1. Connectivity matrices of $84 \times 84$ for the DesikanKilliany atlas (Desikan et al., 2006) and $164 \times 164$ for the Destrieux atlas (Destrieux et al., 2010; Fischl et al., 2004) were constructed. We analyzed 
the reproducibility of these networks weighted with the number, percentage and density of streamlines (Hagmann et al., 2008), SIFTed number of streamlines (Smith et al., 2013), fractional anisotropy (FA), and unweighted binary networks using varying threshold values. The percentage of streamlines was calculated by dividing the number of streamlines with the total number of streamlines within the connectivity matrix and the density of streamlines was calculated by dividing the number of streamlines with the average surface area of the corresponding end-point nodes. No normalization with respect to streamline length was required to account for the seeding bias (Hagmann et al., 2008), as the seeding was performed from the WM-GM interface (Smith et al., 2012). In addition, varying SH orders (from 4 to 12) were used in CSD to study the effect of model complexity on the results. A SH order 12 implied using angular super-resolution, as our data was acquired with 75 gradient orientations (Tournier et al., 2007).

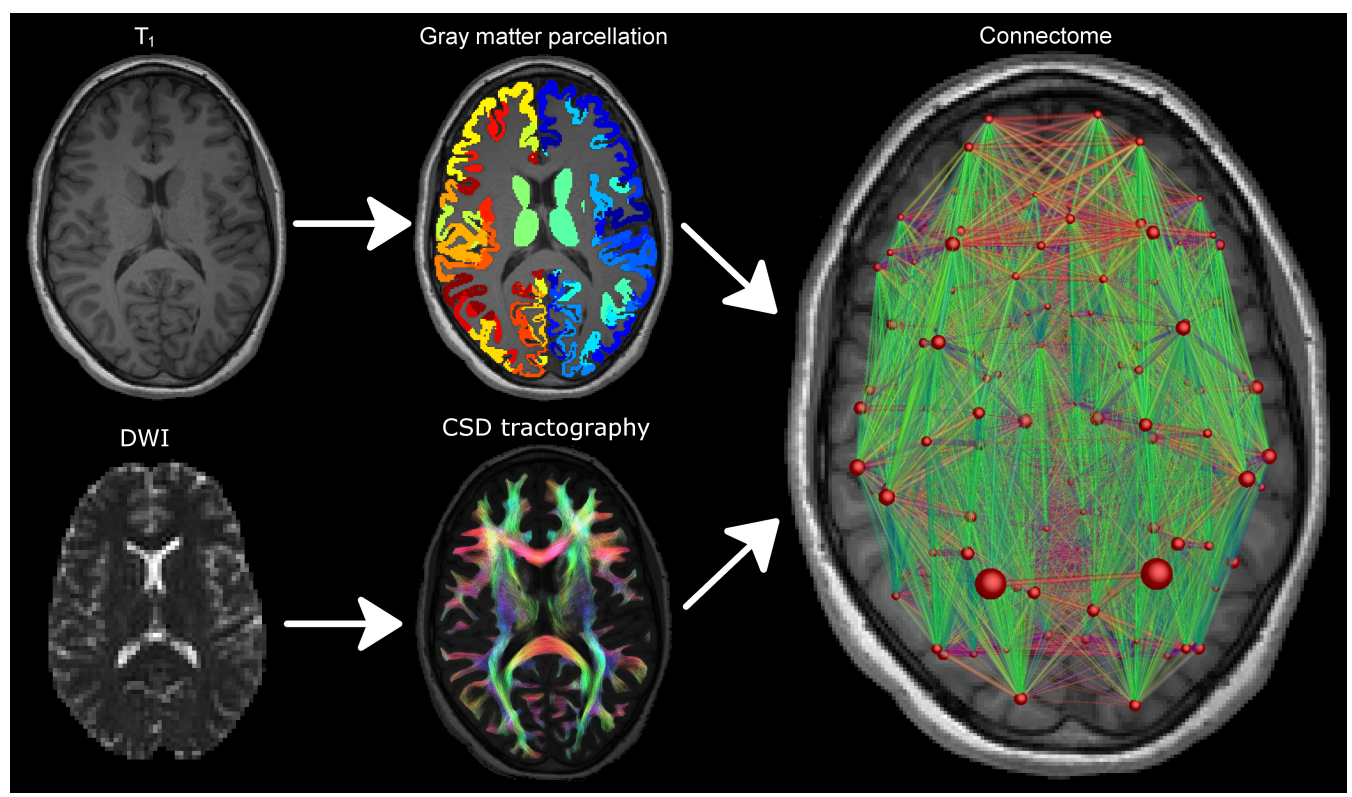

Figure 1: Reconstruction of the structural brain connectome. First, a $\mathrm{T}_{1}$-weighted image was parcellated with FreeSurfer and FSL to segment the cortical and subcortical gray matter structures. Next, whole-brain probabilistic constrained spherical deconvolution (CSD) based streamlines tractography was performed on the motion and distortion corrected diffusion-weighted images (DWI) in MRtrix3. Finally, the structural brain connectome was reconstructed by using the gray matter parcels as the nodes of the network and the streamline tractography results as the edge weights. 
Commonly used graph theoretical metrics were calculated for all of these networks using the Brain Connectivity Toolbox (Bullmore and Sporns, 2009, Rubinov and Sporns, 2010). The investigated metrics were: normalized clustering coefficient (nCC) (Onnela et al., 2005; Saramäki et al., 2007; Watts and Strogatz, 1998), normalized characteristic path length (nCPL) (Watts and Strogatz, 1998), normalized global efficiency (nGE) (Latora and Marchiori, 2001), average local efficiency (LE) (Latora and Marchiori, 2001), betweenness centrality (BC) (Brandes, 2001; Freeman, 1978; Kintali, 2008), small-worldness (SW) (Watts and Strogatz, 1998), and average network degree and strength. Normalization was performed by comparing the networks to 100 randomized networks with equal weight, degree and strength distributions (Rubinov and Sporns, 2011). Local node-level metrics investigated were the clustering coefficient $(\mathrm{CC})$, LE, BC, node degree and strength.

\subsection{Measuring reproducibility by using residual bootstrapping}

Residual bootstrapping with an $8^{\text {th }}$ order $\mathrm{SH}$ representation of the DW signal was performed in each voxel to generate nine additional realizations of each subject's data (Jeurissen et al. 2011), resulting in a total of 190 data sets. These data were then analyzed as described in sections 2.2 2.4 and used to calculate intra-subject variation of the network metrics. Thus, we were able to investigate the reproducibility of the metrics by calculating the intraclass correlation coefficient (ICC) as follows:

$$
\mathrm{ICC}=\frac{\left(\sigma_{\text {inter }}^{2}\right)}{\left(\sigma_{\text {inter }}^{2}+\sigma_{\text {intra }}^{2}\right)},
$$

where $\sigma_{\text {inter }}^{2}$ is the intersubject and $\sigma_{\text {intra }}^{2}$ the intrasubject variance of the same network metric. ICC is the ratio between the intersubject variation and the sum of intersubject and intrasubject variances (Fleiss, 1986; Owen et al., 2013). For example, an ICC higher than 0.75 is traditionally considered excellent reproducibility and values between 0.4 and 0.75 are considered fairto-good (Fleiss, 1986; Owen et al., 2013). In addition, we measured the coefficient of variation of the network metrics by calculating the ratio between intrasubject variance to the mean value of the metric, which allows for the investigation of the relative precision (Vaessen et al., 2010; Owen et al., 2013). 


\section{Results}

\subsection{Reproducibility analyses}

Our results show that the reproducibility of graph theoretical metrics was generally excellent, i.e. the ICC was higher than 0.75 for all metrics both in the residual bootstrapping sample and in the HCP test-retest data. The reproducibility results obtained using a SH order of eight and 10 million reconstructed streamlines, including the corresponding coefficients of variation, are presented in Table 1. In addition, these results indicated that nCPL and SW had the highest coefficients of variation. The reproducibility was generally lower in the HCP test-retest data than in the residual bootstrapping sample, with the exception of $\mathrm{BC}$, but remained excellent for all metrics. Moreover, the intersubject variability of the ICCs in the HCP test-retest data was higher than in the residual bootstrapping sample.

We found that the reproducibility decreased slightly and the variation in the ICCs increased for higher SH orders, i.e. higher model complexities, as shown in Figure 2. However, the reproducibility of all metrics other than BC was excellent for all SH orders. Similar results were obtained in the HCP test-retest sample, as shown in Supplementary Figure 1.

Increasing the reconstruction density gradually from 10,000 to 100,000,000 streamlines improved the reproducibility of both global and local metrics in the residual bootstrapping sample (Figure 3). Excellent reproducibility was reached at approximately one million streamlines, with the exception of local BC values. Nevertheless, the reproducibility continued to increase further up to 10 to 100 million streamlines, especially for local metrics. Only two of the subjects were analyzed with the highest reconstruction density, possibly causing the observed decrease in reproducibility of SW for 100 million streamlines.

In the HCP test-retest data, the reproducibility was generally lower and the standard deviations were clearly higher than in the residual bootstrapping sample, as shown in Figure 4. In addition, the improvement of reproducibility with the reconstruction density was not as prominent. Nevertheless, excellent reproducibility (ICC higher than 0.75 ) of the global metrics was reached at 500,000 to 1 million streamlines, and the reproducibility continued to increase until 10 million streamlines for the local metrics.

In binary networks, reconstructed in the residual bootstrapping sample, the reproducibility of characteristic path length and global efficiency was

significantly decreased for low threshold values of one $(0.00001 \%)$ or ten 
Table 1: Reproducibility of different graph theoretical metrics in networks reconstructed from 10 million streamlines, spherical harmonics order 8 , and weighted by the number of streamlines. The standard deviation of the corresponding value is presented after the \pm sign.

\begin{tabular}{|c|c|c|c|c|}
\hline \multicolumn{5}{|c|}{ Residual bootstrapping } \\
\hline Metric & Intrasubject & Intersubject & $\mathrm{CoV}(\%)$ & ICC \\
\hline $\mathrm{nCC}$ & $1.34 \pm 0.01$ & $1.28 \pm 0.07$ & $0.83 \% \pm 0.44 \%$ & $0.97 \pm 0.05$ \\
\hline $\mathrm{nCPL}$ & $0.43 \pm 0.06$ & $0.49 \pm 0.28$ & $15.93 \% \pm 4.99 \%$ & $0.94 \pm 0.05$ \\
\hline nGE & $0.82 \pm 0.01$ & $0.82 \pm 0.05$ & $4.01 \% \pm 1.45 \%$ & $0.92 \pm 0.05$ \\
\hline LE & $4.44 \mathrm{E}-3 \pm 1.79 \mathrm{E}-4$ & $4.67 \mathrm{E}-3 \pm 6.70 \mathrm{E}-4$ & $4.09 \% \pm 1.74 \%$ & $0.93 \pm 0.05$ \\
\hline $\mathrm{BC}$ & $187 \pm 2.66$ & $185 \pm 5.05$ & $1.42 \% \pm 0.37 \%$ & $0.78 \pm 0.09$ \\
\hline SW & $5.45 \pm 1.01$ & $4.08 \pm 3.46$ & $15.83 \% \pm 4.19 \%$ & $0.91 \pm 0.19$ \\
\hline DG & $100 \pm 1.79$ & $107 \pm 5.55$ & $1.83 \% \pm 0.89 \%$ & $0.90 \pm 0.08$ \\
\hline STR & $70234 \pm 422$ & $72554 \pm 2097$ & $0.60 \% \pm 0.24 \%$ & $0.96 \pm 0.03$ \\
\hline \multicolumn{5}{|c|}{ HCP test-retest dataset } \\
\hline Metric & Intrasubject & Intersubject & $\mathrm{CoV}(\%)$ & ICC \\
\hline $\mathrm{nCC}$ & $1.41 \pm 0.02$ & $1.42 \pm 0.05$ & $1.31 \% \pm 1.09 \%$ & $0.87 \pm 0.15$ \\
\hline $\mathrm{nCPL}$ & $0.35 \pm 0.13$ & $0.37 \pm 0.24$ & $30.8 \% \pm 24.8 \%$ & $0.77 \pm 0.26$ \\
\hline $\mathrm{nGE}$ & $0.80 \pm 0.01$ & $0.80 \pm 0.03$ & $1.76 \% \pm 1.47 \%$ & $0.78 \pm 0.23$ \\
\hline LE & $4.23 \mathrm{E}-3 \pm 2.70 \mathrm{E}-4$ & $4.20 \mathrm{E}-3 \pm 5.10 \mathrm{E}-4$ & $6.44 \% \pm 4.97 \%$ & $0.77 \pm 0.21$ \\
\hline $\mathrm{BC}$ & $176 \pm 2.26$ & $176 \pm 5.33$ & $1.28 \% \pm 1.10 \%$ & $0.84 \pm 0.18$ \\
\hline SW & $5.97 \pm 1.56$ & $5.68 \pm 3.53$ & $30.3 \% \pm 24.4 \%$ & $0.82 \pm 0.20$ \\
\hline DG & $95.8 \pm 1.51$ & $95.6 \pm 4.87$ & $1.58 \% \pm 1.21 \%$ & $0.89 \pm 0.12$ \\
\hline STR & $47844 \pm 566$ & $47684 \pm 1732$ & $1.19 \% \pm 1.01 \%$ & $0.88 \pm 0.14$ \\
\hline
\end{tabular}

CoV: coefficient of variation, ICC: intraclass correlation coefficient, nCC: normalized clustering coefficient, nCPL: normalized characteristic path length, nGE: normalized global efficiency, LE: average local efficiency, BC: betweenness centrality, SW: small-worldness, DG: degree, STR: strength 
$(0.0001 \%)$ streamlines as shown in Figure 5. In networks reconstructed from 10 million streamlines, threshold values of 100 to 1000 streamlines, i.e. $0.01 \%$ to $0.001 \%$, produced the most reproducible results for most of the graph theoretical metrics. For a threshold value of 10,000 streamlines, or $0.1 \%$, the reproducibility of $\mathrm{nCC}, \mathrm{nCPL}, \mathrm{nGE}$ and $\mathrm{SW}$ decreased, although for LE, BC, DG, and STR the reproducibility continued to improve.

The reproducibility results for different network weights are presented in Figure 6 for networks with 10 million streamlines reconstructed with SH order 8 in the residual bootstrapping sample. The reproducibility was excellent for all of the weights and network properties. However, fractional anisotropy weighted and binary networks, especially those without thresholding, had generally lower reproducibility compared to networks weighted with the number, percentage, or density of streamlines. Weighting with the number of SIFT-filtered streamlines resulted in similar reproducibility to the raw number of streamlines, except for the degree and efficiency properties, for which the reproducibility was higher with SIFT. However, although the final reconstruction density was the same as for the other weights, the initial reconstruction density before the application of SIFT was 100 million, which may affect the reproducibility. Moreover, only one of the subjects was processed for intrasubject variability with SIFT.

The reproducibility for the Destrieux atlas (Destrieux et al., 2010; Fischl et al., 2004) with 164 nodes was superior to the Desikan-Killiany atlas (Desikan et al., 2006) with 84 nodes, as shown in Figure 7 in networks with 10 million streamlines, SH order 8 and weighted with the number of streamlines in the residual bootstrapping sample. The effect was similar but less prominent in the HCP test-retest dataset, as shown in Supplementary Figure 2 .

The reproducibility of the local network properties for individual nodes is presented in Figure 8 in networks with 10 million streamlines reconstructed with SH order 8 and weighted with the number of streamlines in the residual bootstrapping sample. The results indicated that the reproducibility of node strength was the highest. The reproducibility of LE was also high, with a similar spatial pattern to $\mathrm{CC}$, for which the reproducibility was slightly lower. The node-level reproducibility was lowest for BC, similar to the global metrics. None of the nodes had an ICC higher than 0.8 for BC, and thus the color scale was changed for this subfigure from 0.8-1.0 to 0.5-1.0. All of the ICC values used in Figure 8 are presented in Supplementary table 1. 
Table 2: Spearman correlation coefficients between different graph theoretical measures in networks reconstructed from 10 million streamlines, spherical harmonics order 8, and weighted by the number of streamlines in the residual bootstrapping sample. The colorcoding describes the correlation coefficients from red (-1) through yellow (0) to green $(+1)$.

\begin{tabular}{|c|c|c|c|c|c|c|c|c|}
\hline Metric & $\mathrm{nCC}$ & nCPL & nGE & LE & BC & sw & DG & STR \\
\hline nCC & & -0.03 & -0.12 & -0.05 & 0.23 & 0.15 & -0.67 & -0.63 \\
\hline nCPL & -0.03 & & -0.20 & -0.02 & -0.31 & -0.99 & 0.15 & 0.11 \\
\hline nGE & -0.12 & -0.20 & & 0.90 & 0.25 & 0.17 & -0.09 & 0.05 \\
\hline LE & -0.05 & -0.02 & 0.90 & & 0.24 & 0.01 & -0.05 & 0.21 \\
\hline BC & 0.23 & -0.31 & 0.25 & 0.24 & & 0.33 & -0.70 & -0.44 \\
\hline SW & 0.15 & -0.99 & 0.17 & 0.01 & 0.33 & & -0.24 & -0.16 \\
\hline DG & -0.67 & 0.15 & -0.09 & -0.05 & -0.70 & -0.24 & & 0.69 \\
\hline STR & -0.63 & 0.11 & 0.05 & 0.21 & -0.44 & -0.16 & 0.69 & \\
\hline
\end{tabular}

nCC: normalized clustering coefficient, nCPL: normalized characteristic path length, nGE: normalized global efficiency, LE: average local efficiency, BC: betweenness centrality, SW: small-worldness, DG: degree, STR: strength

\subsection{Correlation analyses}

Spearman correlation coefficients between the different network metrics in the residual bootstrapping sample are presented in Table 2. The analyses, performed in networks with 10 million reconstructed streamlines and weighted with the number of streamlines, showed that LE correlated strongly with nGE and SW correlated negatively with nCPL. Degree and strength were negatively correlated with $\mathrm{nCC}$ and $\mathrm{BC}$, and positively with each other.

Correlations between network properties from differently weighted networks in the residual bootstrapping sample are presented in Table 3. Spearman correlation coefficients were calculated with respect to the corresponding metric in a network weighted with the number of streamlines. Correlations between the percentage and number of streamlines weighted networks were almost perfect (0.96-1.00) in all of the metrics. Streamline density networks correlated strongly with the number of streamlines networks for $\mathrm{nCC}$, and moderately for nCPL, SW and strength. All of the metrics from the networks weighted with the SIFTed number of streamlines correlated positively $(0.43-$ 0.81 ) with the raw number of streamlines, except for the average strength of the nodes, which had a weak negative correlation. Networks weighted 
Table 3: Spearman correlation coefficients in networks with different weights in the residual bootstrapping sample. The correlation coefficients were calculated with respect to the corresponding metric in a network weighted with the number of streamlines. The networks were reconstructed from 10 million streamlines and a spherical harmonics order 8 . The color-coding describes the correlation coefficients from red (-1) through yellow (0) to green $(+1)$.

\begin{tabular}{|c|c|c|c|c|c|c|c|c|}
\hline Weight & $\mathrm{nCC}$ & nCPL & nGE & LE & BC & sw & DG & STR \\
\hline $\begin{array}{l}\text { Percentage of } \\
\text { streamlines }\end{array}$ & 0.99 & 0.96 & 0.97 & 1.00 & 1.00 & 0.97 & 1.00 & \\
\hline $\begin{array}{l}\text { Density of } \\
\text { streamlines }\end{array}$ & 0.96 & 0.61 & -0.04 & 0.10 & 0.28 & 0.52 & 1.00 & 0.64 \\
\hline $\begin{array}{l}\text { SIFTed } \\
\text { streamlines }\end{array}$ & 0.68 & 0.43 & 0.70 & 0.49 & 0.57 & 0.46 & 0.81 & -0.15 \\
\hline $\begin{array}{l}\text { Fractional } \\
\text { anisotropy }\end{array}$ & 0.84 & 0.31 & -0.37 & -0.41 & 0.70 & 0.26 & 1.00 & 0.65 \\
\hline Binary & 0.79 & -0.08 & -0.18 & -0.13 & 0.70 & 0.20 & 1.00 & 0.69 \\
\hline $\begin{array}{l}\text { Binary } \\
\text { thresholded }\end{array}$ & 0.72 & 0.06 & 0.44 & -0.46 & 0.24 & -0.02 & 0.73 & 0.74 \\
\hline
\end{tabular}

nCC: normalized clustering coefficient, nCPL: normalized characteristic path length, nGE: normalized global efficiency, LE: average local efficiency, BC: betweenness centrality, SW: small-worldness, DG: degree, STR: strength, SIFT: spherical-deconvolution informed filtering of tractograms

with fractional anisotropy correlated strongly with those weighted with the number of streamlines for $\mathrm{nCC}$ and moderately for $\mathrm{BC}$ and strength. A weak negative correlation was observed in the efficiency metrics between the number of streamlines and fractional anisotropy weighted networks. Binary networks correlated with the number of streamlines weighted networks in $\mathrm{nCC}$ and strength, and without thresholding also in BC. Note that due to its binary calculation, the number of adjacent nodes, the degree does not depend on the weight used. In addition, average node strength in networks weighted with the percentage of streamlines is equal for all subjects and equals the degree in binary networks. 


\section{Discussion}

Our results show that the reproducibility of structural brain network properties is excellent both in the residual bootstrapping sample and in the HCP test-retest dataset. These results complement the previous results by Owen et al. (2013) and Smith et al. (2015a), and encourage the investigation of structural brain networks also in clinical samples.

Based on our results, we provide the following guidelines for the reconstruction and analysis of structural brain networks:

- The reconstruction density should be sufficiently high: the absolute lower limit for excellent reproducibility is between 500,000 and one million streamlines (see Figures 3 and 4). However, the reproducibility continues to increase further with increasing number of streamlines (e.g. 10 and 100 million), especially for the local network metrics. To reach these reconstruction densities after SIFT (Smith et al., 2013), 10 to 20 times the desired number of streamlines should initially be reconstructed.

- For reproducible analysis of binary networks, sufficiently high threshold values should be used. The absolute values for the most reproducible results range from 100 to 1000 streamlines in networks with a total number of 10 million streamlines, which equals $0.001 \%$ to $0.01 \%$ of the streamlines (see Figure 5). With lower thresholds, the noiseinduced variation among the nodes with the lowest weights is captured by the binary networks, increasing the variability in the network metrics. However, it has also been discussed that the weak connections may have a significant role in the brain connectivity (Bassett and Bullmore, 2016). More information about the advantages and disadvantages of thresholding the networks is provided in Drakesmith et al. (2015).

- The spherical harmonics order has only a small effect on the reproducibility; higher orders, i.e. more complex models, result in slightly lower reproducibility (see Figure 2). The use of the higher spherical harmonics orders of 8 or more may have other benefits not investigated in this study, such as the ability to resolve sharper crossing angles (Tournier et al., 2008). More details about the recommended acquisition parameters and corresponding spherical harmonics orders for CSD are provided in Tournier et al. (2013). 
- In addition, various network properties correlate strongly with each other, and therefore, it would often be sufficient to study either nCPL or SW, LE or nGE, and degree or strength (see Table 2). This will increase the power of the statistical analyses through the need of smaller correction for multiple comparisons. Between the percentage and number of streamlines weighted networks the correlations were almost perfect (see Table 3). However, correlations between networks with other weights and number of streamlines were much lower, indicating that choosing an appropriate weight makes a substantial difference in the network analyses.

We would like to remind that in addition to the above guidelines, which are directly based on our data, the acquisition and preprocessing of the data should be performed with great care. For instance, the reliable assignment of the streamlines to the corresponding cortical and subcortical GM regions requires proper EPI distortion correction (Andersson et al., 2003; Irfanoglu et al., 2012, Wu et al., 2008) and rigid coregistration (Ashburner and Friston, 2003) between the anatomical and diffusion-weighted data, in addition to eddy current and motion correction (Andersson and Sotiropoulos, 2016, Leemans and Jones, 2009). An excellent pipeline is proposed in Smith et al. (2012).

Moreover, determining biologically accurate measures for structural connectivity based on diffusion MRI data is not unequivocal (Daducci et al. 2016; Jones et al., 2013; Smith et al., 2013, 2015b; Yeh et al., 2016). We showed that the use of the SIFT method to improve the biological correspondence of streamlines (Smith et al., 2013; Yeh et al., 2016) does not decrease the reproducibility in the subsequent graph theoretical analyses, and may improve the reproducibility of the efficiency properties. In addition, we showed that the metrics calculated with and without SIFT differ from each other, as the correlation coefficients were generally below 0.70 (Table 3). However, due to the need for a significantly higher initial number of streamlines and the processing time of the SIFT method, the processing requires approximately 24-48 hours of analysis time per subject on a powerful computer. Nevertheless, the use is recommended in the reconstruction biologically meaningful structural brain networks (Smith et al., 2013, 2015a, b; Yeh et al., 2016).

A limitation of this study was the lack of repeated data acquisition in the original residual bootstrapping sample. Therefore, we replicated the experiments related to the reconstruction density, spherical harmonics order, and 
the two cortical parcellations in the HCP test-retest dataset of 44 healthy subjects. These experiments produced results similar to the residual bootstrapping experiments, and thus, further strengthen the conclusions of the study. The main difference in the results was the lower reproducibility and higher variation in the HCP dataset (except for BC), possibly due to the long interval (4.9 \pm 2.4 months) between the two intrasubject acquisitions and the repositioning of the subjects, which are effects that are not captured by design in residual bootstrapping.

In the future, the non-WM partial volume effects (Roine et al., 2014, 2015a), choices in defining the response function (Parker et al., 2013; Tax et al., 2014), as well as the benefits of multi-shell data (Jeurissen et al., 2014) on the reproducibility of structural brain networks should also be investigated. Especially for the WM-GM interface seeding approach, accounting for the non-WM partial volume effects may have significant benefits.

\section{Conclusions}

We analyzed the reproducibility of structural brain network properties in a residual bootstrapping sample and in the HCP test-retest dataset. Networks were reconstructed with probabilistic CSD-based streamlines tractography while varying several reconstruction and network parameters.

We showed that the reproducibility of structural brain networks reconstructed with CSD is generally excellent. However, streamline density should be sufficient and binarization of the networks should be performed with adequate threshold levels. In addition, certain network properties and connectivity weights correlated with each other, but several connectivity weights did not correlate, which indicates that they may measure different properties. Thus, appropriate connectivity weights should be selected carefully. These guidelines should be taken into account in future research concerning structural brain networks in order to enable reliable investigation of the network properties.

\section{Acknowledgements}

This work was supported by the Fund for Scientific Research-Flanders (FWO), and by the Interuniversity Attraction Poles Program (P7/11) initiated by the Belgian Science Policy Office. In addition, T.R. received support from the Instrumentarium Science Foundation (Finland), the Automation 
Foundation (Finland), the Finnish Foundation for Technology Promotion, and Emil Aaltonen Foundation (Finland). The research of A.L. is supported by VIDI Grant 639.072.411 from the Netherlands Organisation for Scientific Research (NWO). B.J. is a postdoctoral fellow supported by the Research Foundation Flanders (FWO Vlaanderen). We acknowledge the computational resources provided by the Aalto Science-IT project. The authors report no conflicts of interest.

The replication data were provided by the Human Connectome Project, WU-Minn Consortium (Principal Investigators: David Van Essen and Kamil Ugurbil; 1U54MH091657) funded by the 16 NIH Institutes and Centers that support the NIH Blueprint for Neuroscience Research; and by the McDonnell Center for Systems Neuroscience at Washington University.

\section{Vitae}

Timo Roine received his M.Sc.(Tech.) in 2009 from the Helsinki University of Technology (currently Aalto University), Finland. In 2017 he obtained his Ph.D. about diffusion MRI and connectomics from the imec-Vision Lab of the University of Antwerp, Belgium under the supervision of Dr. Ben Jeurissen, Prof. Alexander Leemans and Prof. Jan Sijbers. Before starting his Ph.D., he performed research on mineral processing technology from 2005 to 2010 in the Control Engineering group of Aalto University, Finland, and from 2010 to 2012 he worked as a consultant for healthcare management in Nordic Healthcare Group, Finland. From January 2017 to July 2017 he worked at the departments of Radiology and Psychiatry at the Lausanne University Hospital, Switzerland. Currently, he works as a post-doctoral researcher at the HUS Medical Imaging, Radiology, University of Helsinki, Finland and Turku Brain and Mind Center, University of Turku, Finland.

Ben Jeurissen received an MSc in Computer Science in 2004 and in Biomedical Imaging in 2006, both from the University of Antwerp, Belgium. In 2012, he obtained a PhD in Science from the University of Antwerp. From 2012, he worked as a post-doctoral researcher in the Vision Lab, at the Physics department of the University of Antwerp and at The Florey Institute of Neuroscience and Mental Health in Melbourne, Australia. He is a Junior Fellow of the International Society of Magnetic Resonance in Medicine and specializes in image processing of diffusion MRI data for investigating microstructural tissue organization. 
Daniele Perrone is a post-doctoral researcher in the "Image Processing and Interpretation" research group, at the Department of Telecommunications and Information Processing, Ghent University, Belgium, and part of the Flemish ICT research institute imec. His research focuses on modeling and restoration of Diffusion MR images, with special attention to imaging of brain tissue microstructures and connectivity mapping.

Jan Aelterman is a post-doctoral researcher in the "Image Processing and Interpretation" research group, at the Department of Telecommunications and Information Processing, Ghent University, Belgium. He is currently supported by a Ghent University BOF postdoctoral fellowship (BOF15/PDO/003). His research focuses on restoration of natural images, with an emphasis on (MRI) reconstruction.

In 1989, Wilfried Philips received the Diploma degree in electrical engineering and in 1993 the Ph.D. in applied sciences, both from Ghent University, Belgium. From 1989 to 1997 he worked at the Department of Electronics and Information Systems of Ghent University. Since 1997, he has been at the Department of Telecommunications and Information Processing of Ghent University, where he is currently a full-time professor and is heading the research group "Image Processing and Interpretation", which is also part of the Flemish ICT research institute imec. Wilfried Philips is also a senior member of IEEE.

Jan Sijbers graduated in Physics in 1993. In 1998, he received a $\mathrm{PhD}$ in Physics from the University of Antwerp, for which he received the Scientific Award BARCO NV in 1999. He was an FWO Postdoc at the University of Antwerp and the Delft University of Technology from 2002-2008. In 2005, he became a lecturer and in 2010 senior lecturer. In 2014, he was appointed full professor at the University of Antwerp. His research interests are in biomedical image reconstruction, processing and analysis, focused to magnetic resonance and computed tomography images. He (co-)authored over 250 journal publications. Jan Sijbers is the head of imec-Vision Lab and co-founder of IcoMetrix.

Alexander Leemans is a physicist who received his PhD in 2006 at the University of Antwerp, Belgium. From 2007 to 2009, he worked as a postdoctoral researcher at the Cardiff University Brain Research Imaging Center (CUBRIC), Cardiff University, Wales, United Kingdom. In 2009, he joined the Image Sciences Institute (ISI), University Medical Center Utrecht, the Netherlands, where he currently holds a tenured faculty position as Associate Professor. His current research interests include modeling, processing, visu- 
alizing and analyzing diffusion MRI data for investigating microstructural and architectural tissue organization. He heads the PROVIDI Lab and is the developer of the software ExploreDTI.

\section{References}

Aganj, I., Lenglet, C., Jahanshad, N., Yacoub, E., Harel, N., Thompson, P.M., Sapiro, G., 2011. A hough transform global probabilistic approach to multiple-subject diffusion MRI tractography. Medical Image Analysis $15,414-425$.

Andersson, J.L., Skare, S., Ashburner, J., 2003. How to correct susceptibility distortions in spin-echo echo-planar images: application to diffusion tensor imaging. NeuroImage 20, 870-888.

Andersson, J.L., Sotiropoulos, S.N., 2015. Non-parametric representation and prediction of single-and multi-shell diffusion-weighted MRI data using Gaussian processes. NeuroImage 122, 166-176.

Andersson, J.L., Sotiropoulos, S.N., 2016. An integrated approach to correction for off-resonance effects and subject movement in diffusion MR imaging. NeuroImage 125, 1063-1078.

Andreotti, J., Jann, K., Melie-Garcia, L., Giezendanner, S., Dierks, T., Federspiel, A., 2014. Repeatability analysis of global and local metrics of brain structural networks. Brain connectivity 4, 203-220.

Ashburner, J., Friston, K., 2003. Rigid body registration, in: Frackowiak, R., Friston, K., Frith, C., Dolan, R., Friston, K., Price, C., Zeki, S., Ashburner, J., Penny, W. (Eds.), Human Brain Function. 2nd ed.. London: Academic Press, pp. 635-655.

Basser, P.J., Mattiello, J., LeBihan, D., 1994a. Estimation of the effective self-diffusion tensor from the NMR spin echo. Journal of Magnetic Resonance, Series B 103, 247-254.

Basser, P.J., Mattiello, J., LeBihan, D., 1994b. MR diffusion tensor spectroscopy and imaging. Biophysical Journal 66, 259-267. 
Basser, P.J., Pajevic, S., Pierpaoli, C., Duda, J., Aldroubi, A., 2000. In vivo fiber tractography using DT-MRI data. Magnetic Resonance in Medicine 44, 625-632.

Bassett, D.S., Brown, J.A., Deshpande, V., Carlson, J.M., Grafton, S.T., 2011. Conserved and variable architecture of human white matter connectivity. NeuroImage 54, 1262-1279.

Bassett, D.S., Bullmore, E.T., 2016. Small-world brain networks revisited. The Neuroscientist , 1073858416667720.

Bastiani, M., Shah, N.J., Goebel, R., Roebroeck, A., 2012. Human cortical connectome reconstruction from diffusion weighted MRI: the effect of tractography algorithm. NeuroImage 62, 1732-1749.

Behrens, T., Berg, H.J., Jbabdi, S., Rushworth, M., Woolrich, M., 2007. Probabilistic diffusion tractography with multiple fibre orientations: What can we gain? NeuroImage 34, 144-155.

Brandes, U., 2001. A faster algorithm for betweenness centrality. Journal of mathematical sociology 25, 163-177.

Buchanan, C.R., Pernet, C.R., Gorgolewski, K.J., Storkey, A.J., Bastin, M.E., 2014. Test-retest reliability of structural brain networks from diffusion MRI. NeuroImage 86, 231-243.

Bullmore, E., Sporns, O., 2009. Complex brain networks: graph theoretical analysis of structural and functional systems. Nature Reviews Neuroscience 10, 186-198.

Cheng, H., Wang, Y., Sheng, J., Kronenberger, W.G., Mathews, V.P., Hummer, T.A., Saykin, A.J., 2012. Characteristics and variability of structural networks derived from diffusion tensor imaging. NeuroImage 61, 11531164 .

Daducci, A., Dal Palú, A., Descoteaux, M., Thiran, J.P., 2016. Microstructure informed tractography: pitfalls and open challenges. Frontiers in Neuroscience 10, 247.

Dell'Acqua, F., Scifo, P., Rizzo, G., Catani, M., Simmons, A., Scotti, G., Fazio, F., 2010. A modified damped Richardson-Lucy algorithm to reduce 
isotropic background effects in spherical deconvolution. NeuroImage 49, $1446-1458$.

Dennis, E.L., Jahanshad, N., Toga, A.W., McMahon, K.L., De Zubicaray, G.I., Martin, N.G., Wright, M.J., Thompson, P.M., 2012. Test-retest reliability of graph theory measures of structural brain connectivity, in: International Conference on Medical Image Computing and Computer-Assisted Intervention, Springer. pp. 305-312.

Desikan, R.S., Ségonne, F., Fischl, B., Quinn, B.T., Dickerson, B.C., Blacker, D., Buckner, R.L., Dale, A.M., Maguire, R.P., Hyman, B.T., Albert, M.S., Killiany, R.J., 2006. An automated labeling system for subdividing the human cerebral cortex on MRI scans into gyral based regions of interest. NeuroImage 31, 968-980. doi:http://dx.doi.org/10.1016/j.neuroimage. 2006.01.021.

Destrieux, C., Fischl, B., Dale, A., Halgren, E., 2010. Automatic parcellation of human cortical gyri and sulci using standard anatomical nomenclature. NeuroImage 53, 1-15. doi:http://dx.doi.org/10.1016/j.neuroimage. 2010.06.010.

Dimitriadis, S.I., Drakesmith, M., Bells, S., Parker, G.D., Linden, D.E., Jones, D.K., 2017. Improving the reliability of network metrics in structural brain networks by integrating different network weighting strategies into a single graph. Frontiers in Neuroscience 11, 694.

Drakesmith, M., Caeyenberghs, K., Dutt, A., Zammit, S., Evans, C.J., Reichenberg, A., Lewis, G., David, A.S., Jones, D.K., 2015. Schizophrenialike topological changes in the structural connectome of individuals with subclinical psychotic experiences. Human Brain Mapping 36, 2629-2643.

Duda, J.T., Cook, P.A., Gee, J.C., 2014. Reproducibility of graph metrics of human brain structural networks. Frontiers in neuroinformatics 8, 46.

Farquharson, S., Tournier, J.D., Calamante, F., Fabinyi, G., SchneiderKolsky, M., Jackson, G.D., Connelly, A., 2013. White matter fiber tractography: why we need to move beyond DTI: Clinical article. Journal of Neurosurgery 118, 1367-1377.

Feinberg, D.A., Moeller, S., Smith, S.M., Auerbach, E., Ramanna, S., Glasser, M.F., Miller, K.L., Ugurbil, K., Yacoub, E., 2010. Multiplexed 
echo planar imaging for sub-second whole brain fMRI and fast diffusion imaging. PloS one 5, e15710.

Fischl, B., van der Kouwe, A., Destrieux, C., Halgren, E., Ségonne, F., Salat, D.H., Busa, E., Seidman, L.J., Goldstein, J., Kennedy, D., et al., 2004. Automatically parcellating the human cerebral cortex. Cerebral Cortex $14,11-22$.

Fleiss, J.L., 1986. Reliability of measurement. The design and analysis of clinical experiments , 1-32.

Forde, N.J., O’Donoghue, S., Scanlon, C., Emsell, L., Chaddock, C., Leemans, A., Jeurissen, B., Barker, G.J., Cannon, D.M., Murray, R.M., et al., 2015. Structural brain network analysis in families multiply affected with bipolar I disorder. Psychiatry Research: Neuroimaging 234, 44-51.

Freeman, L.C., 1978. Centrality in social networks conceptual clarification. Social networks 1, 215-239.

Glasser, M.F., Sotiropoulos, S.N., Wilson, J.A., Coalson, T.S., Fischl, B., Andersson, J.L., Xu, J., Jbabdi, S., Webster, M., Polimeni, J.R., et al., 2013. The minimal preprocessing pipelines for the Human Connectome Project. NeuroImage 80, 105-124.

Hagmann, P., Cammoun, L., Gigandet, X., Meuli, R., Honey, C.J., Wedeen, V.J., Sporns, O., 2008. Mapping the structural core of human cerebral cortex. PLoS Biol 6, e159.

van der Horn, H.J., Kok, J.G., de Koning, M.E., Scheenen, M.E., Leemans, A., Spikman, J.M., van der Naalt, J., 2016. Altered wiring of the human structural connectome in adults with mild traumatic brain injury. Journal of Neurotrauma .

Irfanoglu, M.O., Walker, L., Sarlls, J., Marenco, S., Pierpaoli, C., 2012. Effects of image distortions originating from susceptibility variations and concomitant fields on diffusion MRI tractography results. NeuroImage 61, $275-288$.

Jenkinson, M., Bannister, P., Brady, M., Smith, S., 2002. Improved optimization for the robust and accurate linear registration and motion correction of brain images. NeuroImage 17, 825-841. 
Jenkinson, M., Beckmann, C.F., Behrens, T.E., Woolrich, M.W., Smith, S.M., 2012. Fsl. NeuroImage 62, 782-790.

Jeurissen, B., Descoteaux, M., Mori, S., Leemans, A., 2017. Diffusion MRI fiber tractography of the brain. NMR in Biomedicine, e3785.

Jeurissen, B., Leemans, A., Jones, D.K., Tournier, J.D., Sijbers, J., 2011. Probabilistic fiber tracking using the residual bootstrap with constrained spherical deconvolution. Human Brain Mapping 32, 461-479.

Jeurissen, B., Leemans, A., Tournier, J.D., Jones, D.K., Sijbers, J., 2013. Investigating the prevalence of complex fiber configurations in white matter tissue with diffusion magnetic resonance imaging. Human Brain Mapping $34,2747-2766$.

Jeurissen, B., Tournier, J.D., Dhollander, T., Connelly, A., Sijbers, J., 2014. Multi-tissue constrained spherical deconvolution for improved analysis of multi-shell diffusion MRI data. NeuroImage 103, 411-426.

Jones, D.K., Knösche, T.R., Turner, R., 2013. White matter integrity, fiber count, and other fallacies: the do's and don'ts of diffusion MRI. NeuroImage $73,239-254$.

Kintali, S., 2008. Betweenness centrality: Algorithms and lower bounds. arXiv preprint arXiv:0809.1906 .

Kristo, G., Leemans, A., Raemaekers, M., Rutten, G.J., Gelder, B., Ramsey, N.F., 2013. Reliability of two clinically relevant fiber pathways reconstructed with constrained spherical deconvolution. Magnetic Resonance in Medicine 70, 1544-1556.

Latora, V., Marchiori, M., 2001. Efficient behavior of small-world networks. Physical review letters 87, 198701.

Leemans, A., Jones, D.K., 2009. The B-matrix must be rotated when correcting for subject motion in DTI data. Magnetic Resonance in Medicine 61, 1336-1349.

Milchenko, M., Marcus, D., 2013. Obscuring surface anatomy in volumetric imaging data. Neuroinformatics 11, 65-75. 
Moeller, S., Yacoub, E., Olman, C.A., Auerbach, E., Strupp, J., Harel, N., Uğurbil, K., 2010. Multiband multislice GE-EPI at 7 tesla, with 16-fold acceleration using partial parallel imaging with application to high spatial and temporal whole-brain fMRI. Magnetic Resonance in Medicine 63, 1144-1153.

Mugler, J.P., Brookeman, J.R., 1990. Three-dimensional magnetizationprepared rapid gradient-echo imaging (3D MP RAGE). Magnetic Resonance in Medicine 15, 152-157.

Odish, O.F., Caeyenberghs, K., Hosseini, H., Van Den Bogaard, S.J., Roos, R.A., Leemans, A., 2015. Dynamics of the connectome in Huntington's disease: A longitudinal diffusion MRI study. NeuroImage: Clinical 9, 3243.

Onnela, J.P., Saramäki, J., Kertész, J., Kaski, K., 2005. Intensity and coherence of motifs in weighted complex networks. Physical Review E 71, 065103.

Owen, J.P., Ziv, E., Bukshpun, P., Pojman, N., Wakahiro, M., Berman, J.I., Roberts, T.P., Friedman, E.J., Sherr, E.H., Mukherjee, P., 2013. Testretest reliability of computational network measurements derived from the structural connectome of the human brain. Brain connectivity 3, 160-176.

Parker, C.S., Deligianni, F., Cardoso, M.J., Daga, P., Modat, M., Dayan, M., Clark, C.A., Ourselin, S., Clayden, J.D., 2014. Consensus between pipelines in structural brain networks. PloS one 9, e111262.

Parker, G.D., Marshall, D., Rosin, P.L., Drage, N., Richmond, S., Jones, D.K., 2013. A pitfall in the reconstruction of fibre ODFs using spherical deconvolution of diffusion MRI data. NeuroImage 65, 433-448.

Patenaude, B., Smith, S.M., Kennedy, D.N., Jenkinson, M., 2011. A Bayesian model of shape and appearance for subcortical brain segmentation. NeuroImage 56, 907-922.

Rodrigues, P., Prats-Galino, A., Gallardo-Pujol, D., Villoslada, P., Falcon, C., Prčkovska, V., 2013. Evaluating structural connectomics in relation to different q-space sampling techniques, in: International Conference on Medical Image Computing and Computer-Assisted Intervention, Springer. pp. 671-678. 
Roine, T., Jeurissen, B., Perrone, D., Aelterman, J., Leemans, A., Philips, W., Sijbers, J., 2014. Isotropic non-white matter partial volume effects in constrained spherical deconvolution. Frontiers in Neuroinformatics 8, 28. doi:10.3389/fninf.2014.00028.

Roine, T., Jeurissen, B., Perrone, D., Aelterman, J., Philips, W., Leemans, A., Sijbers, J., 2015a. Informed constrained spherical deconvolution (iCSD). Medical Image Analysis 24, 269-281.

Roine, U., Roine, T., Salmi, J., Nieminen-von Wendt, T., Tani, P., Leppämäki, S., Rintahaka, P., Caeyenberghs, K., Leemans, A., Sams, M., 2015b. Abnormal wiring of the connectome in adults with highfunctioning autism spectrum disorder. Molecular Autism 6, 65. doi:10. 1186/s13229-015-0058-4.

Rubinov, M., Sporns, O., 2010. Complex network measures of brain connectivity: uses and interpretations. NeuroImage 52, 1059-1069.

Rubinov, M., Sporns, O., 2011. Weight-conserving characterization of complex functional brain networks. NeuroImage 56, 2068-2079.

Saramäki, J., Kivelä, M., Onnela, J.P., Kaski, K., Kertesz, J., 2007. Generalizations of the clustering coefficient to weighted complex networks. Physical Review E 75, 027105.

Setsompop, K., Gagoski, B.A., Polimeni, J.R., Witzel, T., Wedeen, V.J., Wald, L.L., 2012. Blipped-controlled aliasing in parallel imaging for simultaneous multislice echo planar imaging with reduced g-factor penalty. Magnetic Resonance in Medicine 67, 1210-1224.

Smith, R.E., Tournier, J.D., Calamante, F., Connelly, A., 2012. Anatomically-constrained tractography: Improved diffusion MRI streamlines tractography through effective use of anatomical information. NeuroImage 62, 1924-1938.

Smith, R.E., Tournier, J.D., Calamante, F., Connelly, A., 2013. SIFT: spherical-deconvolution informed filtering of tractograms. NeuroImage 67, 298-312. 
Smith, R.E., Tournier, J.D., Calamante, F., Connelly, A., 2015a. The effects of SIFT on the reproducibility and biological accuracy of the structural connectome. NeuroImage 104, 253-265.

Smith, R.E., Tournier, J.D., Calamante, F., Connelly, A., 2015b. SIFT2: Enabling dense quantitative assessment of brain white matter connectivity using streamlines tractography. NeuroImage 119, 338-351.

Smith, S.M., Jenkinson, M., Woolrich, M.W., Beckmann, C.F., Behrens, T.E., Johansen-Berg, H., Bannister, P.R., De Luca, M., Drobnjak, I., Flitney, D.E., et al., 2004. Advances in functional and structural MR image analysis and implementation as FSL. NeuroImage 23, S208-S219.

Sotiropoulos, S., Moeller, S., Jbabdi, S., Xu, J., Andersson, J., Auerbach, E., Yacoub, E., Feinberg, D., Setsompop, K., Wald, L., et al., 2013a. Effects of image reconstruction on fiber orientation mapping from multichannel diffusion MRI: reducing the noise floor using SENSE. Magnetic Resonance in Medicine 70, 1682-1689.

Sotiropoulos, S.N., Jbabdi, S., Xu, J., Andersson, J.L., Moeller, S., Auerbach, E.J., Glasser, M.F., Hernandez, M., Sapiro, G., Jenkinson, M., Feinberg, D.A., Yacoub, E., Lenglet, C., Essen, D.C.V., Ugurbil, K., Behrens, T.E., 2013b. Advances in diffusion MRI acquisition and processing in the Human Connectome Project. NeuroImage 80, 125-143. doi:http://dx.doi.org/10.1016/j.neuroimage.2013.05.057.

Tax, C.M., Jeurissen, B., Vos, S.B., Viergever, M.A., Leemans, A., 2014. Recursive calibration of the fiber response function for spherical deconvolution of diffusion MRI data. NeuroImage 86, 67-80.

Thompson, D.K., Chen, J., Beare, R., Adamson, C.L., Ellis, R., Ahmadzai, Z.M., Kelly, C.E., Lee, K.J., Zalesky, A., Yang, J.Y., et al., 2016. Structural connectivity relates to perinatal factors and functional impairment at 7years in children born very preterm. NeuroImage 134, 328-337.

Tournier, J., Calamante, F., Connelly, A., 2007. Robust determination of the fibre orientation distribution in diffusion MRI: non-negativity constrained super-resolved spherical deconvolution. NeuroImage 35, 1459-1472.

Tournier, J., Calamante, F., Connelly, A., 2010. Improved probabilistic streamlines tractography by 2nd order integration over fibre orientation 
distributions, in: Proc. 18th Annual Meeting of the Intl. Soc. Mag. Reson. Med.(ISMRM), p. 1670.

Tournier, J., Calamante, F., Connelly, A., 2012. MRtrix: Diffusion tractography in crossing fiber regions. International Journal of Imaging Systems and Technology 22, 53-66.

Tournier, J., Calamante, F., Connelly, A., et al., 2013. Determination of the appropriate $\mathrm{b}$ value and number of gradient directions for high-angularresolution diffusion-weighted imaging. NMR in Biomedicine 26, 1775-1786.

Tournier, J., Calamante, F., Gadian, D.G., Connelly, A., 2004. Direct estimation of the fiber orientation density function from diffusion-weighted MRI data using spherical deconvolution. NeuroImage 23, 1176-1185.

Tournier, J., Yeh, C.H., Calamante, F., Cho, K.H., Connelly, A., Lin, C.P., 2008. Resolving crossing fibres using constrained spherical deconvolution: validation using diffusion-weighted imaging phantom data. NeuroImage $42,617-625$.

Tuch, D.S., 2004. Q-ball imaging. Magnetic Resonance in Medicine 52, $1358-1372$.

Vaessen, M., Hofman, P., Tijssen, H., Aldenkamp, A., Jansen, J., Backes, W.H., 2010. The effect and reproducibility of different clinical DTI gradient sets on small world brain connectivity measures. NeuroImage 51, 11061116 .

Van Essen, D.C., Smith, S.M., Barch, D.M., Behrens, T.E., Yacoub, E., Ugurbil, K., Consortium, W.M.H., et al., 2013. The WU-Minn Human Connectome project: an overview. NeuroImage 80, 62-79.

Vos, S.B., Jones, D.K., Viergever, M.A., Leemans, A., 2011. Partial volume effect as a hidden covariate in DTI analyses. NeuroImage 55, 1566-1576.

Watts, D.J., Strogatz, S.H., 1998. Collective dynamics of small-world networks. Nature 393, 440-442.

Wu, M., Chang, L.C., Walker, L., Lemaitre, H., Barnett, A.S., Marenco, S., Pierpaoli, C., 2008. Comparison of epi distortion correction methods in 
diffusion tensor MRI using a novel framework, in: International Conference on Medical Image Computing and Computer-Assisted Intervention, Springer. pp. 321-329.

Xu, J., Moeller, S., Strupp, J., Auerbach, E., Chen, L., Feinberg, D., Ugurbil, K., Yacoub, E., 2012. Highly accelerated whole brain imaging using aligned-blipped-controlled-aliasing multiband EPI, in: Proceedings of the 20th Annual Meeting of ISMRM, p. 2306.

Yeh, C.H., Smith, R.E., Liang, X., Calamante, F., Connelly, A., 2016. Correction for diffusion MRI fibre tracking biases: The consequences for structural connectomic metrics. NeuroImage 142, 150-162.

Zalesky, A., Fornito, A., Cocchi, L., Gollo, L.L., van den Heuvel, M.P., Breakspear, M., 2016. Connectome sensitivity or specificity: which is more important? NeuroImage 142, 407-420.

Zhong, S., He, Y., Gong, G., 2015. Convergence and divergence across construction methods for human brain white matter networks: an assessment based on individual differences. Human Brain Mapping 36, 1995-2013. 


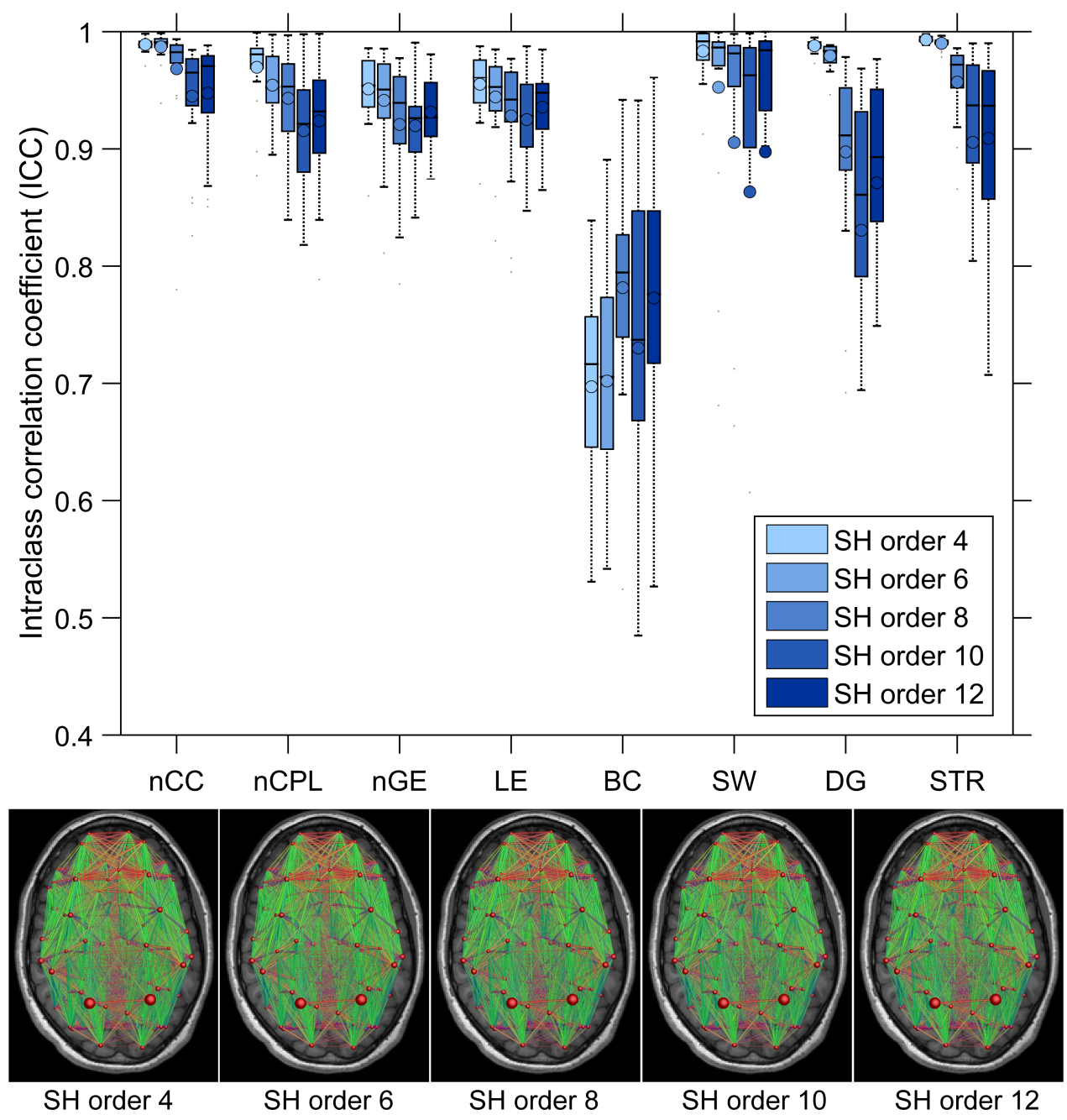

Figure 2: Reproducibility measured with intraclass correlation coefficient (ICC) for different spherical harmonics (SH) orders and graph theoretical measures in the residual bootstrapping sample. Networks were reconstructed from 10 million streamlines and weighted with the number of streamlines. The box plots indicate the mean (circle), median, minimum, maximum, $25^{\text {th }}$ and $75^{\text {th }}$ percentile values. Outliers are indicated with dots. nCC: normalized clustering coefficient, nCPL: normalized characteristic path length, nGE: normalized global efficiency, LE: average local efficiency, BC: betweenness centrality, SW: small-worldness, DG: degree, STR: strength. 

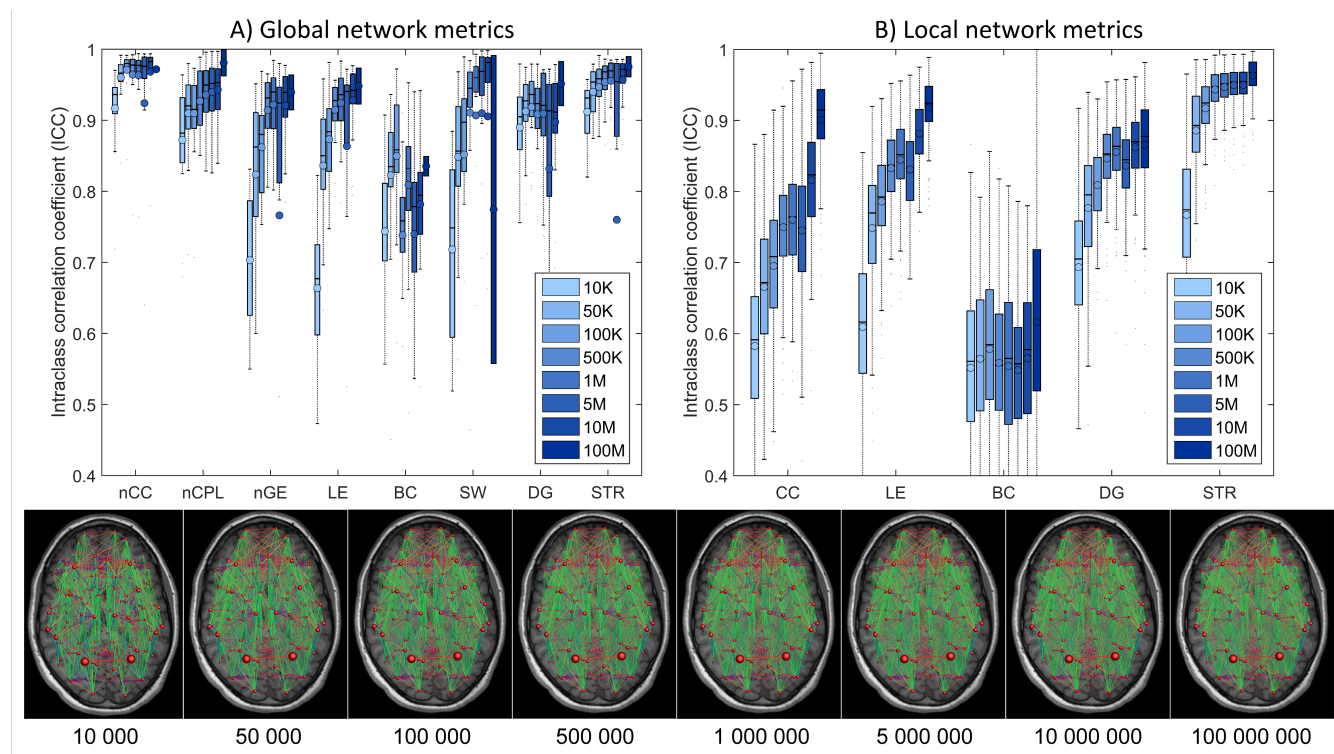

Figure 3: Reproducibility of A) global (nCC: normalized clustering coefficient, nCPL: normalized characteristic path length, nGE: normalized global efficiency, LE: average local efficiency, BC: betweenness centrality, SW: small-worldness, DG: degree, STR: strength) and B) local (CC: clustering coefficient, LE: local efficiency, BC: betweenness centrality, DG: degree, STR: strength) network properties with varying reconstruction densities (total number of streamlines) in the residual bootstrapping sample. The networks were weighted with the percentage of streamlines, and a spherical harmonics order 8 was used. The box plots indicate the mean (circle), median, minimum, maximum, $25^{\text {th }}$ and $75^{\text {th }}$ percentile values. Outliers are indicated with dots. 
A) Global network metrics

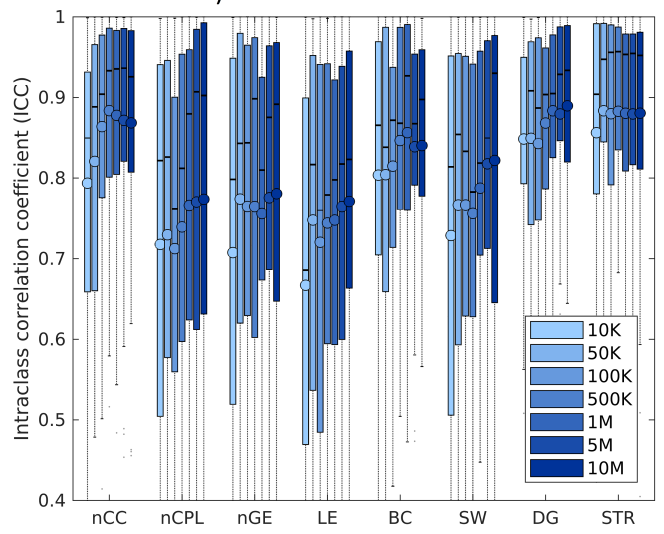

B) Local network metrics

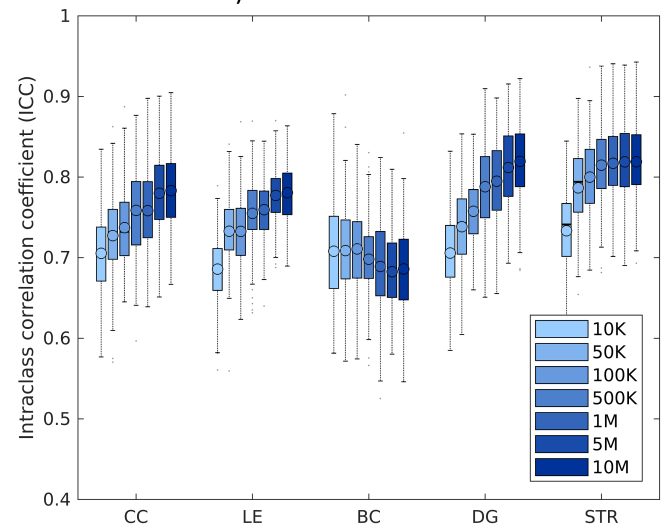

Figure 4: Reproducibility of A) global (nCC: normalized clustering coefficient, nCPL: normalized characteristic path length, nGE: normalized global efficiency, LE: average local efficiency, BC: betweenness centrality, SW: small-worldness, DG: degree, STR: strength) and B) local (CC: clustering coefficient, LE: local efficiency, BC: betweenness centrality, DG: degree, STR: strength) network properties with varying reconstruction densities (total number of streamlines) in the HCP test-retest sample. The networks were weighted with the number of streamlines, and a spherical harmonics order 8 was used. The box plots indicate the mean (circle), median, minimum, maximum, $25^{\text {th }}$ and $75^{\text {th }}$ percentile values. Outliers are indicated with dots. 


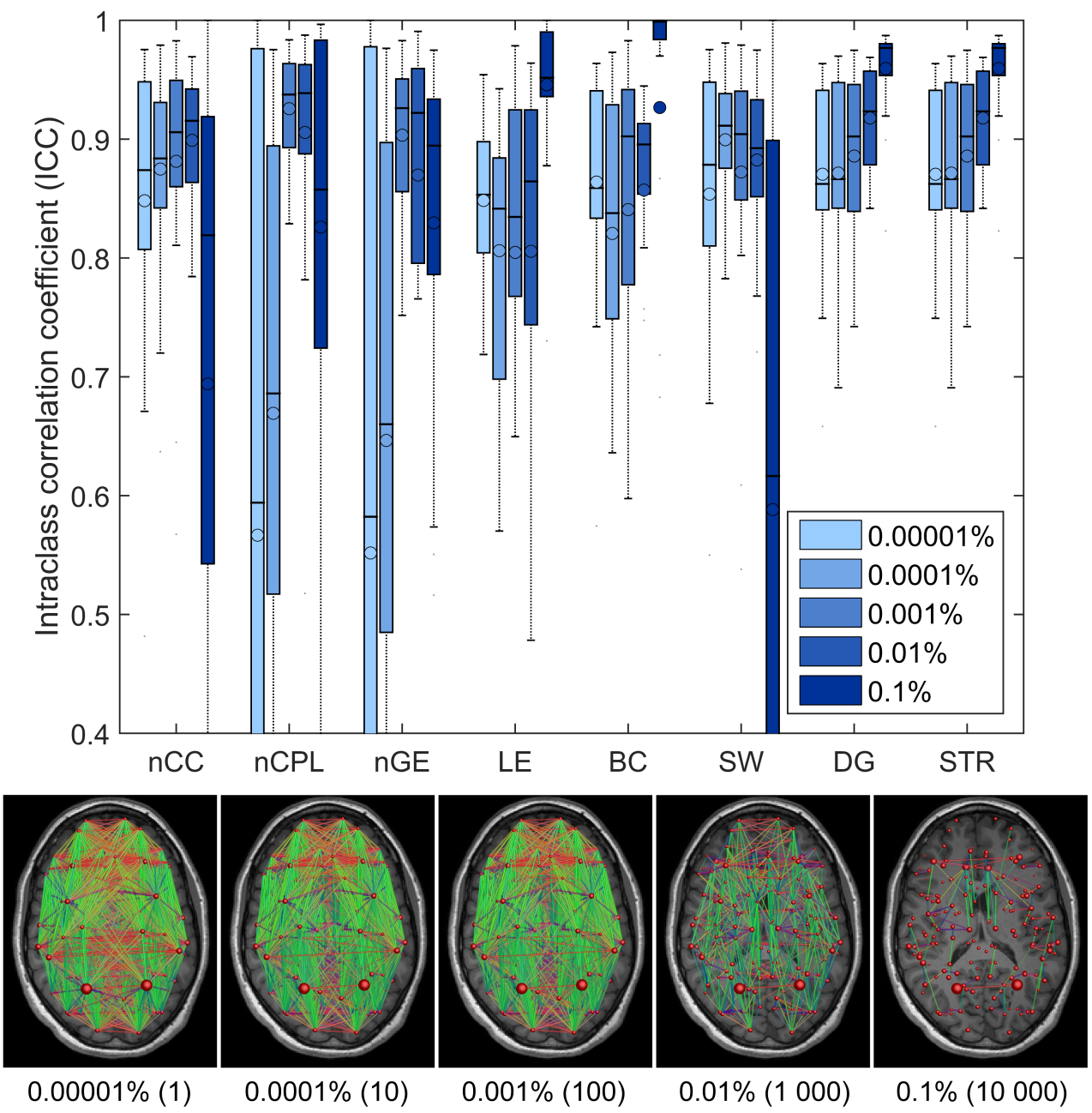

Figure 5: Reproducibility of network properties in binary networks reconstructed from the number of streamlines using varying threshold values in the residual bootstrapping sample. Networks were reconstructed with spherical harmonics ( $\mathrm{SH})$ order 8 and a reconstruction density of 10 million streamlines. The box plots indicate the mean (circle), median, minimum, maximum, $25^{\text {th }}$ and $75^{\text {th }}$ percentile values. Outliers are indicated with dots. nCC: normalized clustering coefficient, nCPL: normalized characteristic path length, nGE: normalized global efficiency, LE: average local efficiency, BC: betweenness centrality, SW: small-worldness, DG: degree, STR: strength 


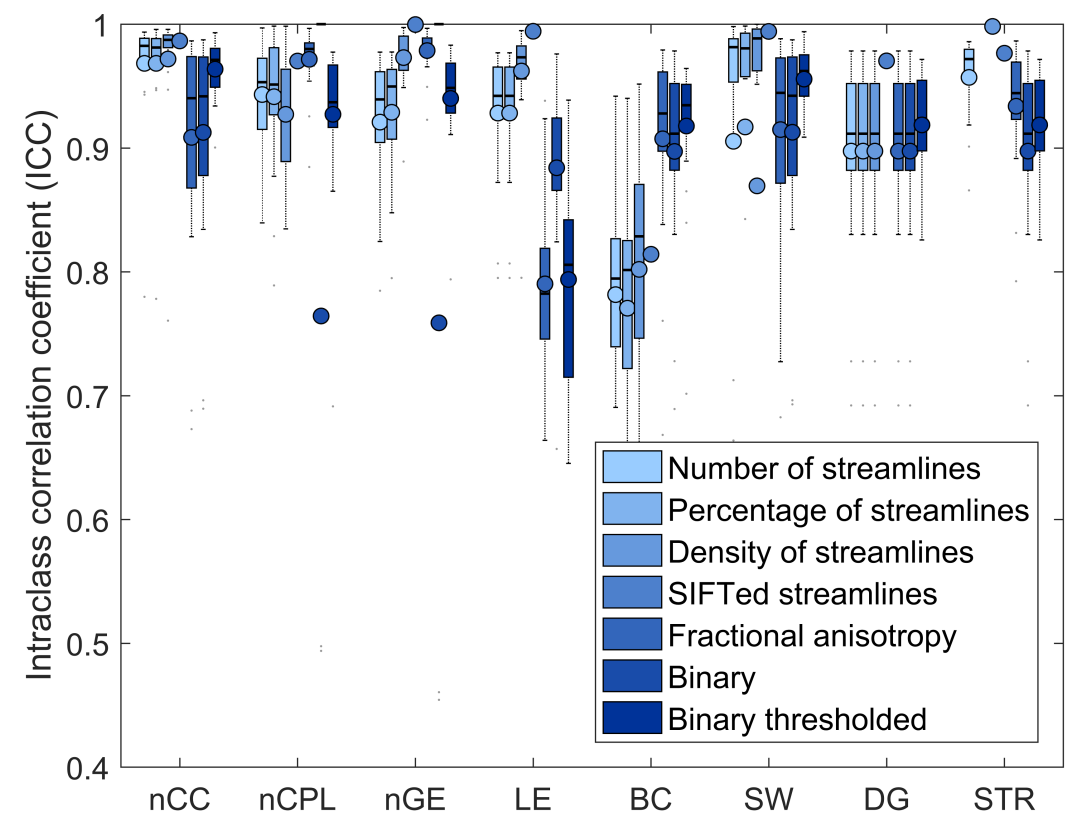

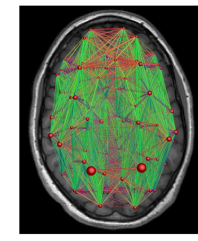

Number of streamlines

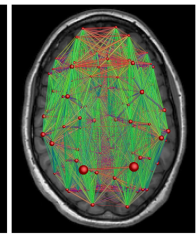

Percentage of streamlines

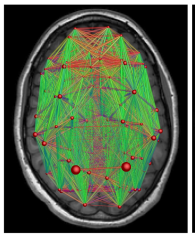

Density of streamlines

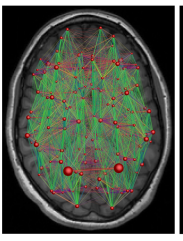

SIFTed streamlines

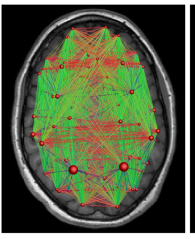

Fractional anisotropy

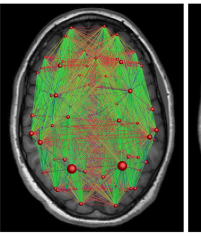

Binary

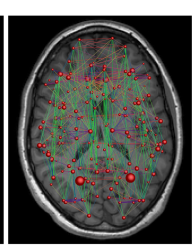

Binary thresholded

Figure 6: Reproducibility of the network properties with different network weights reconstructed from 10 million streamlines and a spherical harmonics (SH) order 8 in the residual bootstrapping sample. The box plots indicate the mean (circle), median, minimum, maximum, $25^{\text {th }}$ and $75^{\text {th }}$ percentile values. Outliers are indicated with dots. nCC: normalized clustering coefficient, nCPL: normalized characteristic path length, nGE: normalized global efficiency, LE: average local efficiency, BC: betweenness centrality, SW: small-worldness, DG: degree, STR: strength, SIFT: spherical-deconvolution informed filtering of tractograms. 


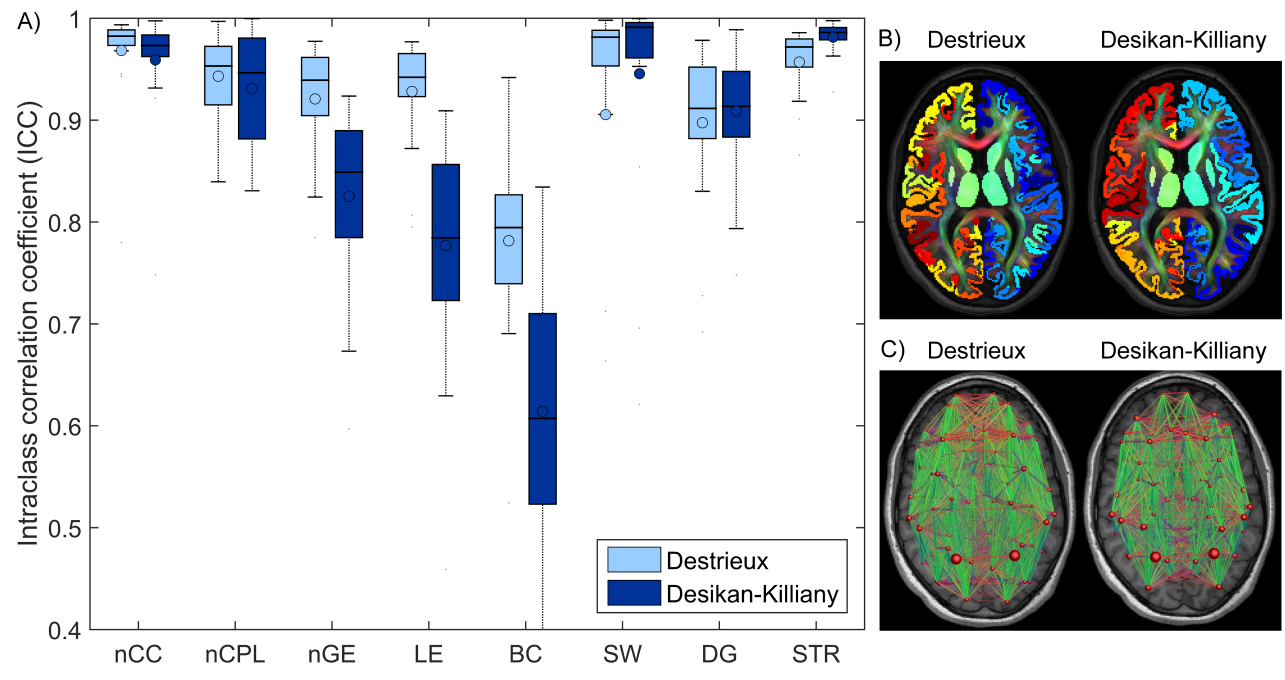

Figure 7: A) Reproducibility of the network properties with two cortical parcellation atlases in FreeSurfer combined with the subcortical structures from FSL's FIRST in the residual bootstrapping sample. Networks were reconstructed from 10 million streamlines and a spherical harmonics ( $\mathrm{SH}$ ) order 8 , and weighted with the number of streamlines. The box plots indicate the mean (circle), median, minimum, maximum, $25^{\text {th }}$ and $75^{\text {th }}$ percentile values. Outliers are indicated with dots. nCC: normalized clustering coefficient, nCPL: normalized characteristic path length, nGE: normalized global efficiency, LE: average local efficiency, BC: betweenness centrality, SW: small-worldness, DG: degree, STR: strength. B) The two atlases (Destrieux: 164 parcels, and Desikan-Killiany: 84 parcels) visualized on an axial slice of the $\mathrm{T}_{1}$-weighted image with the reconstructed streamlines overlaid. C) Visualization of the structural brain networks with the different atlases. The networks were reconstructed as in A). 


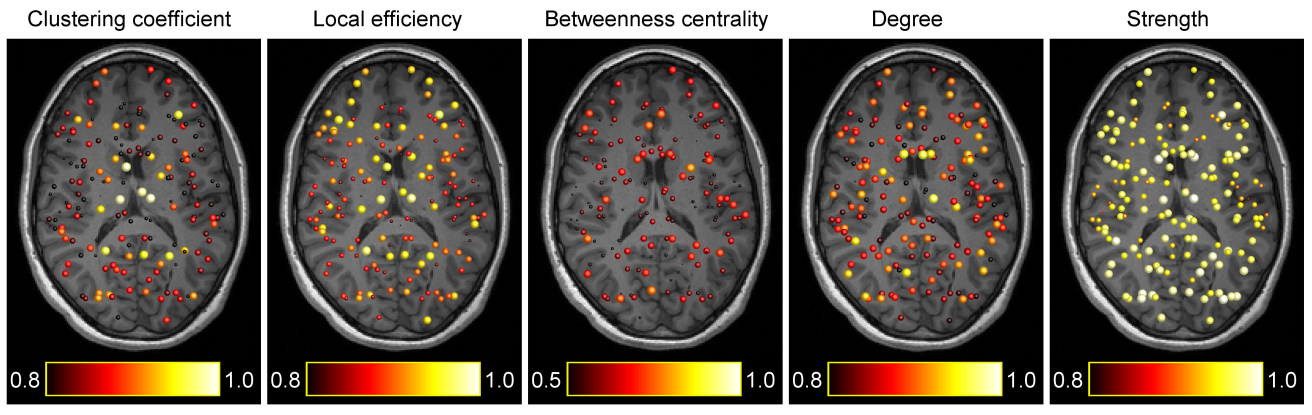

Figure 8: Reproducibility of node-level network properties in networks reconstructed from 10 million streamlines using a spherical harmonics (SH) order 8 and weighted with the number of streamlines in the residual bootstrapping sample. The color of the node indicates the corresponding intraclass correlation coefficient (ICC) according to the scale visualized below, and the size of the node varies from the minimum ICC value to the maximum ICC value within the respective network property. 